\title{
La diffusion spatiale des pièces euro étrangères en Belgique et en France
}

Spatial diffusion of foreign euro coins in Belgium and France

Sandrine Berroir, Claude Grasland, France Guérin-Pace et Grégory Hamez

\section{(2) OpenEdition}

12 Journals

\section{Édition électronique}

URL : http://journals.openedition.org/belgeo/12149

DOI : 10.4000/belgeo.12149

ISSN : 2294-9135

Éditeur :

National Committee of Geography of Belgium, Société Royale Belge de Géographie

\section{Édition imprimée}

Date de publication : 31 décembre 2005

Pagination : 445-458

ISSN : 1377-2368

\section{Référence électronique}

Sandrine Berroir, Claude Grasland, France Guérin-Pace et Grégory Hamez, «La diffusion spatiale des pièces euro étrangères en Belgique et en France », Belgeo [En ligne], 4 | 2005, mis en ligne le 29 octobre 2013, consulté le 01 mai 2019. URL : http://journals.openedition.org/belgeo/12149 ; DOI : 10.4000/belgeo.12149

Ce document a été généré automatiquement le 1 mai 2019.

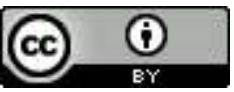

Belgeo est mis à disposition selon les termes de la licence Creative Commons Attribution 4.0 International. 


\section{La diffusion spatiale des pièces euro étrangères en Belgique et en France}

Spatial diffusion of foreign euro coins in Belgium and France

Sandrine Berroir, Claude Grasland, France Guérin-Pace et Grégory Hamez

\section{Introduction}

1 Les frontières intérieures de l'Union européenne sont traversées par des flux croissants de population. Ces flux sont variés en termes de motif et de portée spatiale : il s'agit de mobilité résidentielle à l'échelle internationale, comme le montre l'arrivée massive d'Européens du nord en France (leur nombre a crû de 46 \% de 1999 à 2004) (Borrel et Durr, 2005) ou en Belgique (notamment dans la région de Bruxelles-Capitale) (Hermia et Perrin, 2003); de mobilité résidentielle à l'échelle transfrontalière, d'autant plus forte qu'une grande ville se trouve sur la frontière (Vandermotten et al., 2004); de mobilité transfrontalière pour des motifs de travail, d'achats, de loisirs, de fréquentation d'établissements scolaires ou de santé. En raison de cette diversité thématique et spatiale, il est peu aisé de dessiner le motif d'ensemble de ces flux. Cet article propose un nouvel indicateur pour suivre cette mobilité : l'euro, la nouvelle monnaie commune mise en service en janvier 2002.

2 Les pièces en euro présentent en effet la particularité d'avoir une face nationale; la présence dans un pays d'euros originaires d'un autre pays peut donc être interprétée en première analyse comme un témoignage de l'existence de liens entre les deux pays, pouvant prendre la forme d'une chaîne d'interactions commerciales entre individus. Les cheminements des pièces sont variés et multiples : la pièce peut parvenir à la suite de nombreux ricochets, voire d'allers-retours (Grasland et Guérin-Pace, 2003). L'intérêt est ici de chercher si des régularités se retrouvent dans certaines régions ou au sein de certains groupes sociaux, afin de montrer si certaines frontières ou tronçons de frontières sont plus perméables que d'autres aux réseaux sociaux et spatiaux. 
3 L'étude porte ici sur les euros en France et en Belgique. La frontière franco-belge présente en effet l'intérêt d'alterner sur son tracé des situations de continuité et de discontinuité linguistique, ainsi que socio-économique, de nature à influencer les relations. La première partie présente de façon globale la distribution des euros étrangers dans les deux pays ; la seconde traite de la diffusion des euros français en Belgique et des euros belges en France, à partir d'une modélisation conjointe de l'effet de la distance à la frontière, de la taille du porte-monnaie et de la barrière linguistique.

\section{Les enquêtes de l'ESDO}

Les enquêtes ont été menées dans les deux pays en décembre 2003 par l'ESDO (Euro Spatial Diffusion Observatory : http://www.esdo.prd.fr).

Les cinq premières enquêtes trimestrielles réalisées en France depuis mars 2002 montrent un effet important des caractéristiques des territoires mais aussi des individus sur la diffusion des euros étrangers. Ainsi, la localisation à proximité d'une frontière, dans une ville ou encore la profession exercée, favorisent l'entrée en contact et la circulation des euros étrangers sur le territoire.

La représentativité géographique de l'échantillon est assurée au niveau des régions de sondage : 5 en Belgique (régions Nielsen) et 9 en France (les 8 ZEAT, avec découpage en deux du Bassin parisien). La taille des échantillons impose le choix de ce découpage territorial assez grossier qui va évidemment exercer une influence importante sur l'analyse des phénomènes d'interaction (Laurent \& Thomas, 1997).

Figure 1. Définition des zones géographiques de sondage.

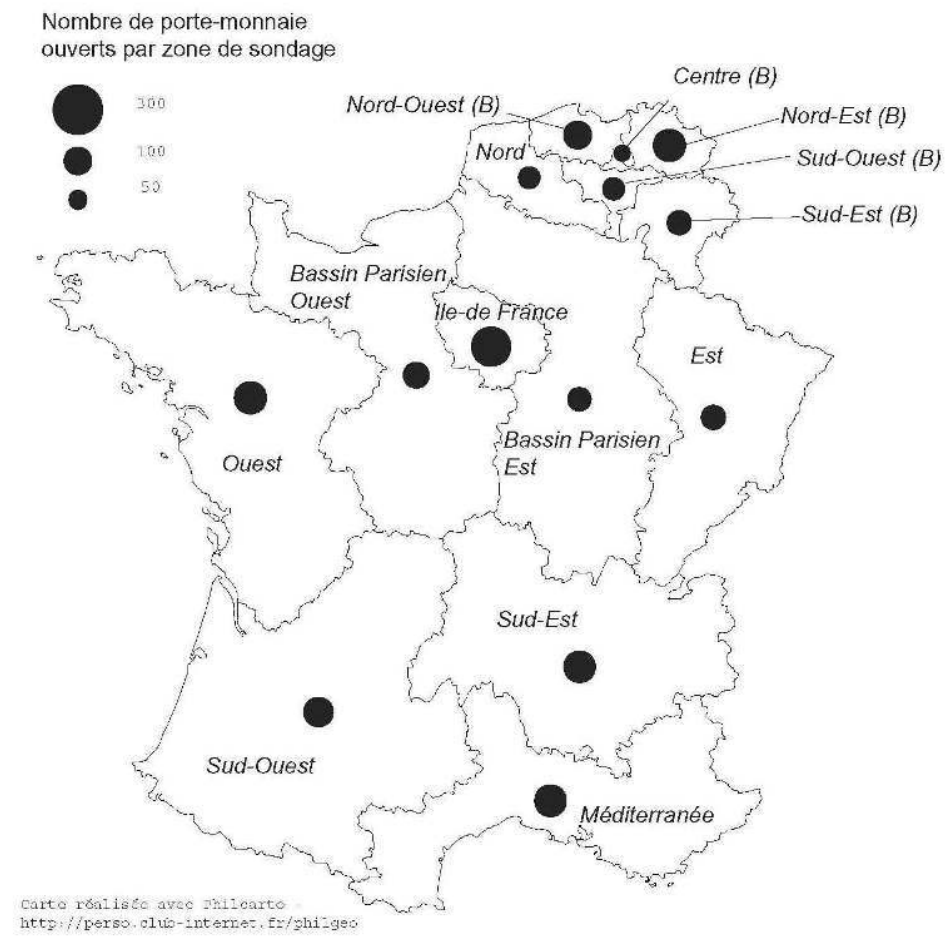


Tableau 1. Caractéristiques principales des enquêtes ESDO.

\begin{tabular}{|c|cccccccc|c|}
\hline & \multicolumn{7}{c}{ France } & Belgique \\
Dates & Mars 02 & Juin 02 & Sept. 02 & Janv. 03 & Juin 03 & Sept. 03 & Dec. 03 & Dec. 03 \\
\hline Nombre de personnes interrogées & 1002 & 2012 & 2057 & 2042 & 1998 & 1978 & 2013 & 977 \\
\% de porte-monnaie observés & 71.6 & 73.7 & 69.5 & 71.9 & 64.6 & 71.1 & 77.3 & 67.9 \\
$\begin{array}{c}\text { Nombre de pièces observées } \\
\text { \% de pièces étrangères dans }\end{array}$ & 10369 & 21395 & 21254 & 19023 & 18460 & 18374 & 21479 & 9769 \\
$\begin{array}{c}\text { l'ensemble des pièces observées } \\
\text { \% de porte-monnaie contenant } \\
\text { au moins une pièce étrangère }\end{array}$ & 4.7 & 6.0 & 9.2 & 11.0 & 11.0 & 13.7 & 14.0 & 26.4 \\
\end{tabular}

\section{La diffusion des euros étrangers en France et en Belgique}

4 En décembre 2003, les pièces étrangères sont nettement plus présentes dans les portemonnaie belges que dans les porte-monnaie français. La probabilité de trouver un euro étranger ${ }^{1}$ dans son porte-monnaie est en effet de 77 \% en Belgique contre $63 \%$ en France.

\section{La répartition régionale des euros étrangers en France et en Belgique}

5 Les variations spatiales de présence des euros étrangers en France et en Belgique combinent plusieurs composantes (Figure 2). La première, certainement la plus structurante dans la propagation des euros, est liée à la proximité d'une frontière. La persistance de cet effet avait déjà été notée dans de précédents travaux (Grasland, Guérin-Pace, Tostain, 2002). Cependant, le rôle des frontières dans la diffusion des euros étrangers est très inégal suivant l'origine des pièces. Alors que cet effet est particulièrement actif pour les pièces émises dans les pays limitrophes, il n'est pas perceptible pour les pièces étrangères de pays non frontaliers. 
Figure 2. Présence des euros étrangers en France et en Belgique.

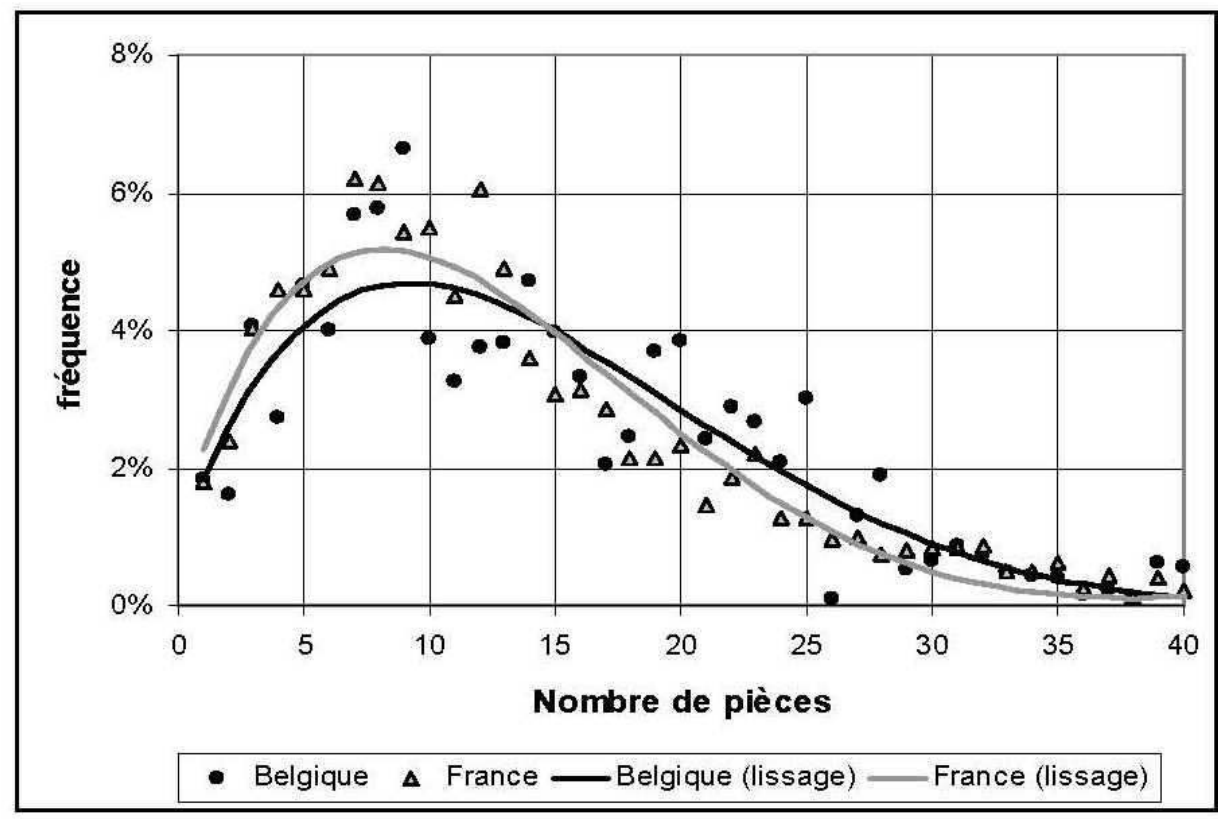

Par ailleurs, le jeu de cette proximité est très variable suivant les frontières. Les frontières France/Belgique, France/Allemagne, France/Luxembourg ou Belgique/Luxembourg semblent particulièrement perméables à la circulation des euros et les zones situées de part et d'autre de ces frontières enregistrent les probabilités les plus élevées d'avoir un euro étranger (Régions Nord et Est du côté français, Brabant wallon et région Sud-est du côté belge). Plus on s'éloigne de ces frontières, plus la probabilité de trouver un euro étranger diminue, tant en Belgique qu'en France. Au contraire, les frontières francoespagnoles ou franco-italiennes semblent moins actives et les zones françaises situées à la frontière de ces pays ne sont pas marquées par une aussi forte présence de pièces étrangères.

7 La deuxième composante, visible à cette échelle macro-régionale, permet de cerner un effet que l'on peut qualifier de métropolitain. En effet, les régions capitales des deux pays (Bruxelles, Paris) apparaissent bien comme des lieux privilégiés. La présence des euros étrangers y est toujours plus élevée que dans les régions environnantes pourtant plus proches des frontières repérées comme "actives» précédemment (par exemple la probabilité d'avoir une pièce étrangère en Ile-de-France (69\%) est plus forte que dans le Bassin parisien est $(44 \%)$ ). C'est sans surprise que ces grandes régions métropolitaines définissent des lieux d'intenses brassages de tous ordres, professionnels, commerciaux, touristiques, etc. La forte présence d'euros étrangers ne fait que traduire cette forme de mobilité des hommes en provenance et à destination de ces grandes régions urbaines.

Enfin, la variabilité de la répartition des euros étrangers est à rapprocher de l'inégale attractivité touristique des régions. En France, c'est à ce facteur que l'on peut certainement imputer la valeur relativement élevée de la probabilité d'avoir un euro étranger dans la région méditerranéenne ou à l'inverse la valeur relativement faible dans la région du Bassin parisien est. 


\section{Les euros étrangers suivant le pays d'origine}

9 Si l'on considère que la circulation des euros peut être un indicateur de l'intensité des échanges qui se nouent entre différents lieux, la comparaison de la présence des euros étrangers suivant le pays d'origine met en évidence des relations inter-étatiques relativement différentes en France et en Belgique (Figure 3).

Figure 3. Probabilité d'avoir au moins un euro étranger dans son porte-monnaie, par pays d'origine.

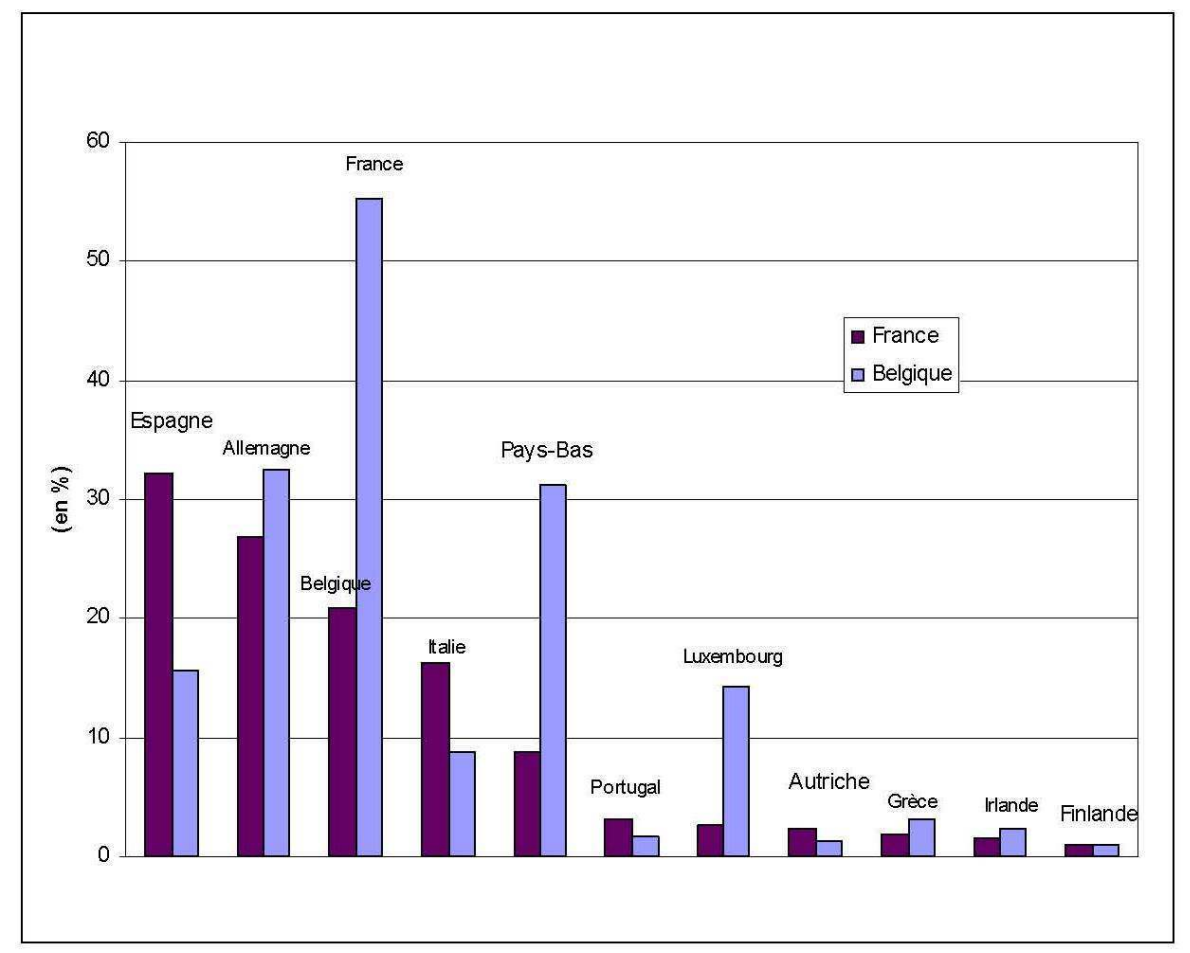

En Belgique, on retiendra en premier lieu la présence très dominante des pièces françaises parmi les pièces étrangères, témoin de l'intensité et de la variété des échanges entre les deux pays. En effet, la probabilité de trouver un euro français dans un portemonnaie belge est de plus de $55 \%$, alors que celle de trouver un euro belge dans les portemonnaie français est de $21 \%$ seulement. Les euros allemands ou néerlandais sont présents dans environ un porte-monnaie belge sur trois, ce qui est nettement moins élevé que les euros français, mais peut sans doute s'expliquer, soit par la taille plus faible du pays émetteur (Pays-Bas), soit par la longueur plus réduite de la frontière (Allemagne). Un troisième groupe est constitué par les euros luxembourgeois et espagnols, présents dans environ un porte-monnaie belge sur sept (14 et $15 \%$ ), et dont l'égale présence montre bien les compensations qui peuvent s'opérer entre la proximité (Luxembourg) et la population du foyer émetteur (Espagne) en matière de diffusion. Ce raisonnement de type "gravitaire » a ses limites si l'on observe que les euros en provenance d'Italie, pays plus peuplé que l'Espagne et plus proche en distance kilométrique, ne se retrouvent que dans moins d'un dixième des porte-monnaie belges. Il faut nécessairement faire intervenir ici d'autres facteurs tels que les relations économiques et surtout touristiques qui interviennent entre les régions. Les autres pays de la zone euro, à la fois petits et éloignés, ont une présence beaucoup plus anecdotique dans les porte-monnaie belges. 
11 En France, la répartition des pièces étrangères selon l'origine est sensiblement différente. $\mathrm{Au}$ delà des variations quantitatives, expression générale de la plus faible présence des pièces étrangères, le classement des pays donné par la présence relative de leur monnaie traduit d'autres relations privilégiées de la France avec les pays européens limitrophes. A travers cet indicateur, c'est d'abord la force des relations avec l'Espagne qui est mise en évidence. En effet, les pièces espagnoles ont environ une chance sur trois de se trouver dans un porte-monnaie français (32\%); elles sont suivies par les pièces allemandes, belges et italiennes qui ont respectivement une chance sur quatre, une chance sur cinq et une chance sur six d'y être présentes. Seule ensuite la présence des euros néerlandais peut encore être notée, alors que comme en Belgique, la présence des autres monnaies européennes devient nettement plus anecdotique.

12 La surreprésentation relative des euros espagnols que l'on observe en France et en Belgique pourrait s'expliquer, outre le facteur touristique, par des effets de corridor de transport sur le territoire français au cours de trajets entre l'Europe du Nord et la péninsule ibérique. Un camionneur anglais, néerlandais ou allemand se rendant au Portugal peut ainsi jouer un rôle de vecteur dans le transport de pièces espagnoles sur le territoire français voire sur le territoire belge. Cette situation est en revanche moins probable dans le cas des euros italiens qui transitent dans des corridors situés plus à l'est permettant d'atteindre les grands ports néerlandais sans passer par la France ou la Belgique.

\section{Des effets de frontière et métropolitains variables suivant l'origine des euros}

13 Globalement l'effet de la proximité d'une frontière joue un rôle sur la diffusion des différentes pièces étrangères, mais agit de manière différenciée suivant l'origine des euros. Ainsi, pour les euros allemands, l'effet frontalier est marqué, tant du côté belge que français (on retrouve les valeurs les plus élevées dans la région Est de la France et dans la région sud-est de la Belgique), et le gradient en fonction de la distance à la frontière allemande est relativement comparable pour les deux pays. Dans le cas des euros italiens, la situation est un peu différente avec une diffusion faiblement différentiée suivant les régions, et une décroissance en fonction de l'éloignement à la frontière franco-italienne beaucoup moins rapide que ne l'est celle observée à partir de la frontière francoallemande. Dernier exemple, celui des euros luxembourgeois: du côté français, la diminution de la présence de ces pièces est particulièrement rapide dès qu'on s'éloigne de la frontière (on passe de $60 \%$ pour les régions limitrophes à $6 \%$ pour les régions les plus proches mais non contiguës à la frontière). La diffusion des euros luxembourgeois sur le territoire français est donc de très courte portée, en lien vraisemblablement avec l'intensité du travail frontalier. Du côté belge, la pénétration des euros luxembourgeois est moins contrastée : les valeurs sont certes plus élevées à proximité de la frontière, mais elles diminuent également moins rapidement, signe d'une plus large diffusion à l'échelle de l'ensemble du territoire belge.

De même, l'effet métropolitain sur la présence des euros étrangers, repérable dans la majorité des cas, ne se traduit pas toujours avec la même intensité. Ainsi, la métropole parisienne apparaît comme particulièrement "attractive", relativement aux autres régions françaises, pour les euros italiens et néerlandais ${ }^{2}$. La spécificité bruxelloise ressort moins nettement, excepté à travers la surreprésentation des euros 
luxembourgeois, sans doute liée aux nombreux échanges entre les pôles des institutions européennes. En revanche, pour les euros allemands ou espagnols en Belgique, on ne peut parler d'effet bruxellois.

\section{Le chassé croisé des euros français et belges}

En nous centrant dans cette seconde partie sur la diffusion spatiale des euros belges en France et des euros français en Belgique, nous allons essayer d'affiner l'analyse des mécanismes de diffusion spatiale et de mieux identifier le rôle de la frontière nationale dans ce processus. Pour ce faire, nous nous appuierons sur un modèle d'interaction spatiale et sur l'interprétation d'éventuels effets résiduels.

\section{Modélisation conjointe des effets de la distance à la frontière et de la taille du porte-monnaie}

16 Le modèle retenu est un modèle logistique qui explique la probabilité de présence d'au moins un euro étranger du pays voisin (p) par la distance entre son lieu de résidence et la frontière la plus proche du pays voisin (DIS) et le nombre de pièces contenues dans le porte-monnaie de l'individu (NBP).

En effet, sachant que la taille du porte-monnaie détermine fortement la probabilité d'avoir une pièce étrangère et que des variations régionales non négligeables existent dans la taille moyenne des porte-monnaie en France comme en Belgique, nous avons choisi d'estimer simultanément ces deux effets. En effet, si nous ne tenions compte que de l'effet de la distance, nous risquerions d'inclure dans les résidus les variations régionales de la taille du porte-monnaie. Par ailleurs, faute de disposer de la localisation exacte des individus, la distance est estimée par celle observée entre la frontière et le centre de l'unité spatiale la plus précise dont nous disposions pour localiser les personnes, soit le niveau NUTS 3 (départements français et arrondissements belges). L'imprécision introduite par cette approximation est peu gênante pour les départements éloignés de la frontière mais elle fausse légèrement les résultats pour les individus situés dans les départements frontaliers. On voit par ailleurs sur la Figure 4 que l'information disponible à ce niveau est nettement moins dense d'un point de vue spatial en France qu'en Belgique, ce qui signifie que la modélisation de la variation de la présence des pièces en fonction de la distance à la frontière sera beaucoup moins précise dans le cas français que dans le cas belge. 
Figure 4. Distribution des porte-monnaie ouverts au niveau NUTS 3.

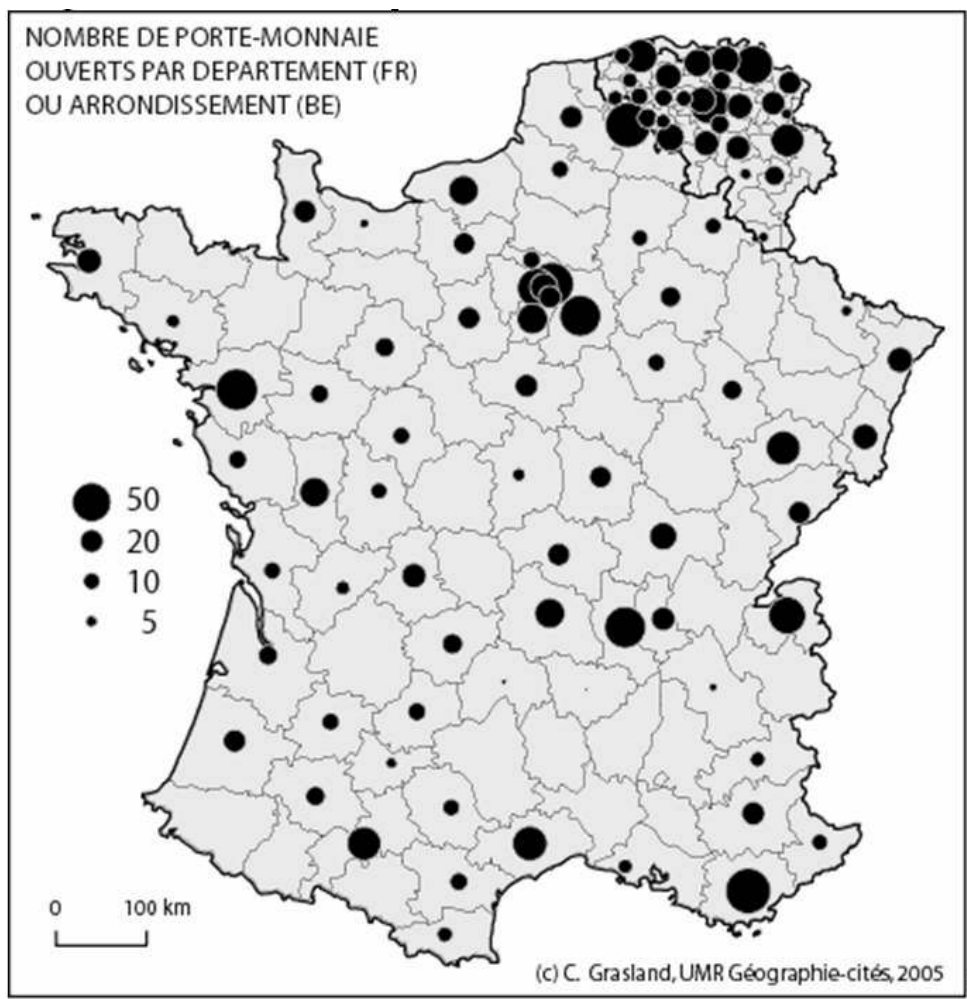

La forme de la relation liant la présence d'une pièce étrangère aux deux variables explicatives a fait l'objet de tests. D'après ces derniers, les fonctions de type puissance semblent plus adaptées que les fonctions exponentielles pour décrire cette relation, qui peut s'écrire sous la forme suivante :

$\log \left(\frac{p}{1-p}\right)=\alpha+\beta \log (N B P)+\gamma \log (D S)+C s t e$

L'estimation des trois paramètres du modèle par la méthode du maximum de vraisemblance permet de déduire pour chaque individu une estimation de sa probabilité de posséder au moins un euro du pays voisin, en fonction du niveau général de présence des euros de ce pays $(\alpha)$, de l'accroissement de la probabilité avec la taille du portemonnaie ( $\beta$ ) et de la décroissance de la probabilité avec la distance au pays émetteur $(\gamma)$. Cette probabilité individuelle se déduit de l'équation précédente et s'écrit :

$$
p=\frac{\boldsymbol{e}^{\alpha}-(N B P)^{\rho}-(D S)^{\boldsymbol{\gamma}}}{1+\left[\varepsilon^{\boldsymbol{\alpha}}-(N B P)^{\boldsymbol{\rho}}-(D S)^{\boldsymbol{\gamma}}\right]}
$$

L'analyse des résultats du modèle montre que les effets des deux variables introduites sont très significatifs. Toutes choses égales quant à la présence des euros du pays voisin, la taille du porte-monnaie semble influer plus fortement sur la probabilité d'avoir un euro belge en France $(\beta=+0.74)$ que sur la probabilité d'avoir un euro français en Belgique $(\beta+0.56)$. Inversement, l'effet négatif de la distance à la frontière semble plus marqué pour la diffusion des euros français en Belgique $(\gamma=-0.85)$ que pour la diffusion des euros belges en France $(\gamma=-0.74)$. Nous verrons par la suite que ce résultat est en partie incomplet car 
imputable à l'effet de barrière linguistique entre la Flandre et la Wallonie, effet non pris en compte dans ce premier modèle.

21 La prise en compte de ces deux grands déterminants qui peuvent être considérés comme «mécaniques» de la présence des euros étrangers - la taille du porte-monnaie et la distance à la frontière - ne constitue qu'une première approche et doit être comprise comme un point de départ pour identifier d'autres facteurs de nature sociale ou territoriale. Nous allons nous centrer sur ces derniers en identifiant l'existence d'effets " régionaux ", indépendants de la distance à la frontière et de la taille des porte-monnaie. L'objectif poursuivi est de repérer les régions belges (ou françaises) pour lesquelles apparaissent des excédents ou des déficits de présence de pièces françaises (ou belges), pouvant être interprétés en termes de relations préférentielles ou d'effets de barrière.

\section{La diffusion des euros français en Belgique : effet de proximité, barrière linguistique}

22 A l'échelle des cinq régions belges de sondage, on constate des écarts importants entre les probabilités, observées et attendues, de posséder des euros d'origine française (Tableau 2).

Tableau 2. Résidus régionaux du modèle de diffusion des euros français en Belgique.

\begin{tabular}{|c|c|c|c|c|c|c|c|c|c|}
\hline Régions & $\begin{array}{c}\text { Effectif } \\
\text { total }\end{array}$ & $\begin{array}{l}\text { Effectif } \\
\text { observé }\end{array}$ & $\begin{array}{l}\text { Effectif } \\
\text { estimé }\end{array}$ & \begin{tabular}{|l} 
Effectif \\
observé/ \\
effectif \\
total \\
\end{tabular} & $\begin{array}{l}\text { Effectif } \\
\text { estimé/ } \\
\text { effectif } \\
\text { total }\end{array}$ & $\begin{array}{l}\text { Effectif } \\
\text { observé/ } \\
\text { effectif } \\
\text { estimé }\end{array}$ & chi2 & $\mathrm{Pr}>\mathrm{HO}$ & \\
\hline I Nord-Ouest & 156 & 84 & 93 & $54 \%$ & $60 \%$ & 0.90 & -0.85 & 0.36 & \\
\hline II Nord Est & 205 & 74 & 91 & $36 \%$ & $44 \%$ & 0.81 & -3.20 & 0.07 & ** \\
\hline III Centre & 57 & 34 & 27 & $60 \%$ & $47 \%$ & 1.29 & 2.18 & 0.14 & * \\
\hline IV Sud Ouest & 103 & 76 & 77 & $74 \%$ & $75 \%$ & 0.98 & -0.02 & 0.88 & \\
\hline V Sud Est & 120 & 85 & 65 & $71 \%$ & $55 \%$ & 1.30 & 5.92 & 0.02 & *** \\
\hline Belgique & 641 & 354 & 354 & $55 \%$ & $55 \%$ & 1.00 & - & - & \\
\hline
\end{tabular}

Résidus significatifs au seuil de : ***: $0.05 ; * *: 0.10 ; *: 0.20$

Effectif total : nombre d'individus ayant accepté de montrer le contenu de leur porte-monnaie. Effectif observé : individus ayant au moins un euro français dans leur porte-monnaie.

Toutes choses égales quant à la taille de leur porte-monnaie et à leur éloignement de la frontière, les personnes situées dans la région sud-est et la région centrale se caractérisent par une nette sur-représentation de la possession d'euros français $(+30 \%)$. Inversement, on observe un déficit d'euros français dans les deux régions flamandes du nord-ouest $(-10 \%)$ et du nord-est (-20\%). La région sud-ouest est quant à elle conforme aux prévisions du modèle. Ces résidus régionaux sont toutefois à considérer avec précaution du fait de la relative faiblesse des échantillons de personnes interrogées.

La surreprésentation des euros français dans la région Sud-Est renvoie sans doute au rôle particulier joué par le Luxembourg: l'intensité du travail frontalier en provenance de France et de Belgique à destination du Grand Duché peut déjà favoriser la circulation des euros entre les trois pays. À cela s'ajoute la fiscalité très favorable du Luxembourg pour des produits souvent achetés en espèces, comme les cigarettes. Enfin, la situation géographique particulière de cette région dite « des trois frontières" rend possible des stratégies originales de la part de la population. Ainsi, des Français viennent résider en 
Belgique, profitant du coût moins élevé de la construction et des terrains, tout en étant travailleurs frontaliers au Luxembourg et en se ravitaillant en France pour certains produits (les Français représentent $4 \%$ de la population de l'arrondissement belge d'Arlon).

Expliquer la faiblesse relative de la présence des euros français dans les régions du nordest et du nord-ouest de la Belgique requiert de prendre en compte la barrière linguistique et ses effets sur les réseaux sociaux de relations qui assurent la circulation des pièces. Toutefois, les tests statistiques effectués sur les résidus ne semblent pas totalement probants. En réalité, ce problème vient du fait que le territoire flamand est en moyenne plus éloigné de la France que la Wallonie, de sorte que le paramètre de frein de la distance a " capturé » une partie de l'effet de barrière. Si l'on veut estimer sans biais l'effet de la limite linguistique sur la diffusion des euros belges en France, il convient donc de l'introduire dans le modèle logistique, simultanément avec l'effet de distance. Nous avons donc pris en compte, dans le modèle logistique, en plus de la distance et de la taille du porte-monnaie, deux variables booléennes mesurant respectivement les effets de la localisation en Flandre ou à Bruxelles (la Wallonie servant de référence). Si l'effet bruxellois n'apparaît pas significatif (la probabilité de trouver un euro français à Bruxelles n'est pas significativement différente de celle de la Wallonie), l'effet Flandre est très significatif et se traduit en moyenne par une nette réduction de la probabilité d'avoir un euro français, surtout lorsqu'on s'éloigne de la frontière. Nous avons tracé sur la Figure 5 la décroissance de la probabilité d'avoir un euro étranger en fonction de la distance à la frontière en Flandre et en Wallonie dans le cas d'un porte-monnaie de référence fixé à 15 pièces.

Figure 5. Décroissance de la probabilité d'avoir un euro français en Belgique en fonction de la zone linguistique (pour un porte-monnaie de 15 pièces).

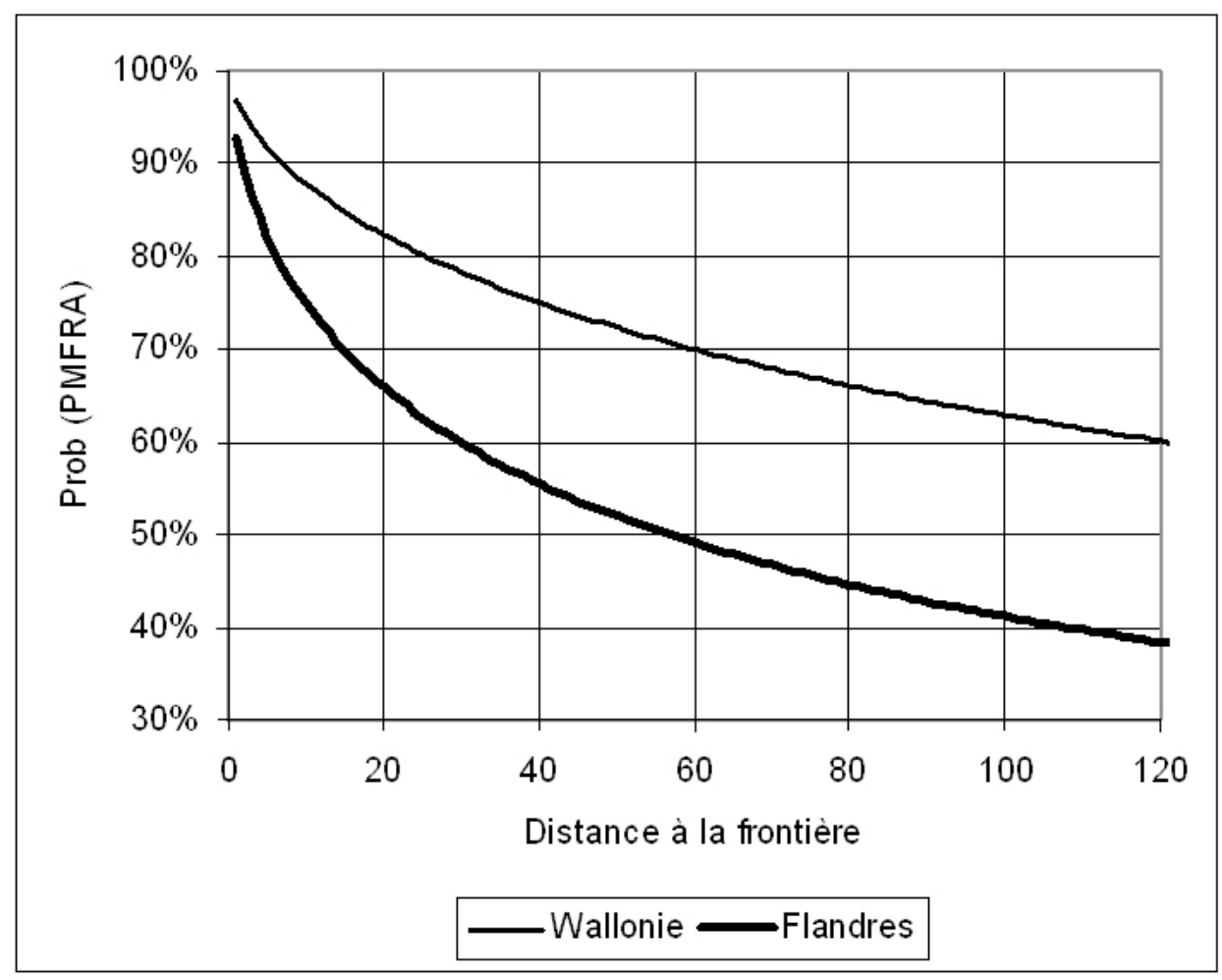


La répartition spatiale des euros français en Belgique reproduit en partie les comportements de mobilité selon une double logique de proximité spatiale et de barrière linguistique (Klaassen et al., 1972 ; Rossera, 1990). En effet, la probabilité d'avoir un euro français dans son porte-monnaie est toujours plus forte à proximité de la frontière, mais elle l'est moins à la limite entre la France et la Flandre. Si globalement, la probabilité d'avoir un euro français décroît avec la distance à la frontière, les arrondissements wallons et flamands ont des profils bien différents : les euros français sont surreprésentés dans les premiers et sous-représentés dans les seconds par rapport à ce que laisserait attendre leur distance à la frontière. Ainsi, dans la banlieue de Bruxelles, la probabilité d'avoir des euros français est particulièrement faible dans l'arrondissement flamand de Halle-Vilvoorde et forte dans l'arrondissement wallon de Nivelles. Cette répartition spatiale est cohérente avec d'autres indicateurs de la présence française, comme le nombre de Français résidant en Belgique (Figure 6). En effet, la présence de ressortissants français décroit également avec la distance à la frontière, et est moins importante en Flandre. Par rapport à la moyenne belge, les Français sont surreprésentés dans les arrondissements frontaliers wallons proches de Lille et proches du Luxembourg, ainsi qu'à Bruxelles.

Figure 6. Les Français en Belgique.

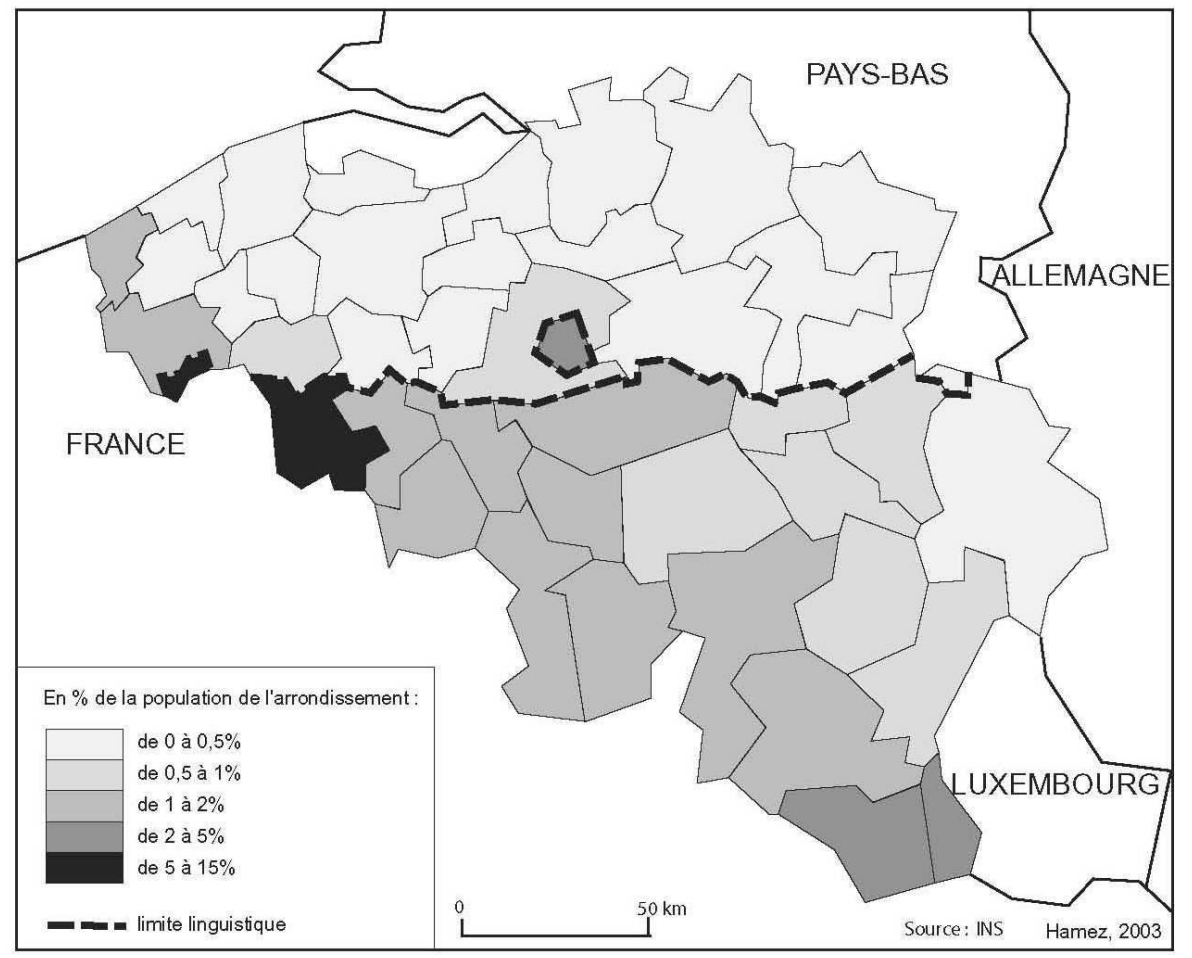

\section{La diffusion des euros belges en France : effet de proximité, tourisme et réseaux de transport}

A première vue, la diffusion spatiale des euros belges sur le territoire français semble obéir à une logique classique de décroissance de la probabilité en fonction de la distance à la frontière belge. Si les euros étrangers sont globalement surreprésentés dans la région 
métropolitaine d'Ile-de-France, il n'en va pas de même pour les euros belges. Comme dans le cas de la Belgique, il n'existe pas d'effet «capitale». Toutes choses égales quant à la taille de leur porte monnaie et leur distance à la frontière, les franciliens n'ont pas une probabilité plus élevée de détenir des euros belges que les autres habitants de la France. La circulation des euros belges se distingue donc de celle des autres euros étrangers qui, rappelons-le, sont surreprésentés en Ile-de-France. Ce résultat va dans le même sens que l'étude d'autres indicateurs. Ainsi, une étude sur la nuptialité mixte entre Français et Européens du Nord, considérée comme un indicateur de l'intensité des relations entre populations, a révélé également que l'Ile-de-France ne connaît pas de surreprésentation particulière pour la part des couples franco-belges, à la différence d'autres couples comme les couples franco-allemands (Hamez, 2004). La présence des Belges sur le territoire français aurait comme spécificité une large diffusion dans toutes les régions, sans prédilection particulière pour l'Ile-de-France.

Il existe, comme dans le cas de la Belgique, un certain nombre de résidus régionaux par rapport au modèle initial (Tableau 3).

Tableau 3. Résidus régionaux du modèle de diffusion des euros belges en France.

\begin{tabular}{|c|c|c|c|c|c|c|c|c|c|}
\hline Régions & $\begin{array}{c}\text { Effectif } \\
\text { total }\end{array}$ & $\begin{array}{l}\text { Effectif } \\
\text { observé }\end{array}$ & $\begin{array}{l}\text { Effectif } \\
\text { estimé }\end{array}$ & $\begin{array}{l}\text { Effectif } \\
\text { observé } \\
\text { effectif } \\
\text { total }\end{array}$ & $\begin{array}{l}\text { Effectif } \\
\text { estimé/ } \\
\text { effectif } \\
\text { total }\end{array}$ & $\begin{array}{l}\text { Effectif } \\
\text { observé/ } \\
\text { effectif } \\
\text { estimé }\end{array}$ & chi2 & $\mathrm{Pr}>\mathrm{H} 0$ & \\
\hline 1 Région Parisienne & 301 & 74 & 75 & $25 \%$ & $25 \%$ & 0.99 & -0.01 & 0.92 & \\
\hline 2 Bassin Parisien Est & 114 & 18 & 30 & $16 \%$ & $26 \%$ & 0.60 & -4.86 & 0.03 & $* * *$ \\
\hline 3 Bassin Parisien Ouest & 136 & 25 & 25 & $18 \%$ & $19 \%$ & 0.97 & -0.02 & 0.88 & \\
\hline 4 Nord & 99 & 74 & 68 & $75 \%$ & $69 \%$ & 1.09 & 0.58 & 0.45 & \\
\hline 5 Est & 121 & 20 & 26 & $17 \%$ & $21 \%$ & 0.77 & -1.33 & 0.25 & \\
\hline 6 Ouest & 206 & 36 & 28 & $17 \%$ & $13 \%$ & 1.29 & 2.28 & 0.13 & * \\
\hline 7 Sud Ouest & 174 & 26 & 20 & $15 \%$ & $12 \%$ & 1.29 & 1.76 & 0.19 & * \\
\hline 8 Sud Est & 202 & 24 & 28 & $12 \%$ & $14 \%$ & 0.88 & -0.41 & 0.52 & \\
\hline 9 Méditerranée & 197 & 24 & 21 & $12 \%$ & $11 \%$ & 1.13 & 0.34 & 0.56 & \\
\hline France & 1550 & 321 & 321 & $21 \%$ & $21 \%$ & 1.00 & - & - & \\
\hline
\end{tabular}

Effectif total : nombre d'individus ayant accepté de montrer le contenu de leur porte-monnaie. Effectif observé : individus ayant au moins un euro belge dans leur porte-monnaie.

Le résidu le plus significatif concerne le Bassin parisien Est où la présence des euros belges est inférieure de $40 \%$ à ce qu'auraient pu laisser prévoir la distance à la frontière et la taille des porte-monnaie des habitants. On retrouve ici une sous- représentation conforme à celle déjà mise en évidence pour les euros étrangers toutes origines confondues. A un moindre degré, les régions de l'Est et du Sud-Est français connaissent également un déficit d'euros belges (-23\% et $-12 \%$ ), mais l'effectif de porte-monnaie ne permet pas d'en démontrer la significativité. A l'inverse, on note une nette surreprésentation des euros belges dans l'Ouest et le Sud-Ouest (+29\%) ainsi que, mais à un moindre degré, dans la région Nord ( $+9 \%)$ et la région Méditerranée $(+12 \%)$. Toutefois, là encore, l'effectif ne permet pas de garantir la significativité des écarts (sauf à accepter un risque d'erreur de $20 \%$ ). S'il ne s'agit pas d'une fluctuation aléatoire, cette diffusion préférentielle des euros belges en France dans l'Ouest et le Sud-Ouest pourrait sans doute être liée aux fréquentatios touristiques du littoral. 
Toujours à titre d'hypothèse, on peut se demander si les itinéraires de déplacement touristiques et professionnels des Belges (mais aussi des Anglais ou des Néerlandais) qui se rendent en France mais aussi en Espagne ou au Portugal ne pourraient pas contribuer à expliquer certaines des sur-représentation observées dans l'ouest et le sud-ouest de la France. Un routier ou un touriste qui se déplace de Bruxelles à Madrid ou Lisbonne a de fortes chances de s'arrêter une ou plusieurs fois le long des aires d'autoroutes qui jalonnent leur itinéraire et d'y effectuer quelques transactions commerciales (achat d'un café, d'un sandwich) qui laisseront une trace spatiale sous la forme d'euros belges à Orléans, Tours, Poitiers ou Bordeaux. Pour valider une telle hypothèse il faudrait procéder à des enquêtes sur la présence des euros étrangers le long des grands axes routiers afin de vérifier si ces derniers contribuent à la constitution de pics locaux de présence des pièces étrangères.

\section{Conclusion}

31 La diffusion des euros en France et en Belgique apporte un nouvel éclairage sur la mobilité internationale. À côté des facteurs «classiques» que sont la distance à la frontière ou l'intensité de la fréquentation touristique, la diffusion est également fonction du facteur linguistique, comme le montre la sous-représentation des euros français dans la Région flamande. Ce résultat rejoint les analyses menées sur la sociabilité transfrontalière en termes de mariages franco-flamands et franco-wallons (Hamez, 2004) mais l'étude de la distribution des euros ajoute un élément supplémentaire: si les mariages renseignent sur la fréquentation interpersonnelle, les euros apportent un éclairage sur la fréquentation des espaces à travers l'ensemble des formes de mobilité, dès lors qu'elles se traduisent par des transactions commerciales mobilisant des échanges de pièces. Quel que soit l'indicateur retenu, la limite linguistique ressort comme un puissant frein aux relations transfrontalières.

Les enquêtes ESDO menées dans le cadre franco-belge montrent toute la richesse de l'objet « euro » comme indicateur de la mobilité mais aussi l'intérêt d'étendre ce type d'enquêtes à d'autres pays, ainsi que plus localement à des zones frontalières. Contrairement à ce que l'on prévoyait au moment de la mise en place de l'euro, au début de l'année 2002, le mouvement de brassage des pièces est beaucoup plus lent que prévu et il serait encore temps de mettre en place une enquête à l'échelle de la zone euro pour un coût raisonnable au regard de l'intérêt des résultats attendus. La crise actuelle de grippe aviaire ne montre-t-elle pas le caractère hautement stratégique pour l'Europe d'une meilleure connaissance des processus de diffusion internationaux?

\section{BIBLIOGRAPHIE}

BORREL C., DURR J.-M. (2005), « Enquêtes annuelles de recensement : premiers résultats de la collecte 2004 ", Insee Première, 1001. 
GRASLAND C., GUÉRIN-PACE F. (2003), « Mobilité européenne, tourisme et diffusion des pièces euros étrangères en France ", XXXIX Colloque de l'ASRDLF, Lyon 1-3 septembre 2003, http:// www.asrdlf2003.entpe.fr.

GRASLAND C., GUÉRIN-PACE F., TOSTAIN A. (2002), « La circulation des euros, reflet de la mobilité des hommes ", Population \& Sociétés, 384, 4 p.

GRASLAND C., MATHIAN H., VINCENT, J.-M. (2000), « Multiscalar analysis and map generalisation of discrete social phenomena: Statistical problems and political consequences », Statistical Journal of the United Nations, ECE, 17, IOS Press, 2, 2000, pp. 1-32.

HAMEZ G. (2004), Du transfrontalier au transnational : Approche géographique. L'exemple de la frontière franco-belge, thèse, Univ. Paris 1.

http://tel.ccsd.cnrs.fr/documents/archives0/00/00/71/91/index_fr.html.

HERMIA J.-P., PERRIN N. (2003), « L'implantation des ressortissants européens à Bruxelles : des logiques migratoires originales ? ", in EGGERICKX T., PERRIN N., Élargissement de l'Union européenne : enjeux et implications socio-démographiques, Louvain-la-Neuve, Chaire Quetelet 2003, thème " Migrations internationales ».

KLAASSEN L.H., WAGENAAR S., WEG A. (1972), « Measuring psychological distance between the Flemings and the Wallons », Papers of the Regional Science Association, 29, pp. 45-62.

LAURENT M.A., THOMAS I. (1997), « Modèle d'interaction spatiale et agrégation des lieux. L'exemple des données criminelles », L'Espace Géographique, 26, 3, pp. 269-279.

ROSSERA F. (1990), Discontinuities in communications among communities of different language in Switzerland, Paper presented at ESF/NECTAR joint worshops « Barriers in communication », Athens, 17-21 February 1990, NETCOM, 4, 1, pp. 119-131.

VANDERMOTTEN C. et al. (2004), Migrations in Europe : The four last decades, Rome, Società Geografica italiana.

\section{NOTES}

1. Ces proportions sont calculées sans prendre en compte les pièces de 50 centimes : en effet, la France a mis en circulation 100 millions de pièces espagnoles de 50 centimes dès le début 2002, ce qui rend ce type de pièces non représentatif de la mobilité.

2. Dire que Paris est «attractive " pour les euros de ces pays ne doit pas préjuger de la direction des flux qui apportent les euros de ces pays. Les pièces peuvent aussi bien être véhiculées par des Italiens ou des Néerlandais effectuant un déplacement à Paris que par des Parisiens se rendant aux Pays-Bas ou en Italie (sans parler des formes plus complexes de transit et de ricochet par des espaces tiers). 


\section{RÉSUMÉS}

Au début de l'année 2002, une nouvelle monnaie, l'euro, a été introduite dans 12 des 15 pays de l'Union Européenne. L'étude de l'origine nationale des pièces peut - dans certaines conditions être utilisée pour produire une image globale de la mobilité des européens à travers les frontières nationales. L'article présente les résultats de deux enquêtes réalisées en France et en Belgique en décembre 2003 sur des échantillons représentatifs de la population. Les résultats obtenus démontrent que la répartition des euros étrangers est fortement liée tout d'abord aux règles habituelles de l'interaction spatiale (distance à la frontière, taille du pays d'origine) mais est également influencée par des facteurs moins triviaux tels que le tourisme ou les barrières linguistiques.

At the beginning of 2002, the new euro currency was introduced in 12 of the 15 States of the European Union. The study of the national origin of coins can be used - under certain conditions - to produce a picture of global mobility of Europeans across national boundaries. The paper presents the results of two surveys carried out in France and Belgium in December 2003 on a representative panel of population. The results demonstrate that the distribution of foreign euros is strongly related to classic spatial interaction rules (distance to boundary, size of the state of origin) but is also influenced by other factors like tourism or linguistic boundaries.

\section{INDEX}

Mots-clés : euro, mobilité internationale, diffusion spatiale, frontière, interaction spatiale, France, Belgique

Keywords : international mobility, spatial diffusion, boundary, spatial interaction, Belgium

\section{AUTEURS}

\section{SANDRINE BERROIR}

UMR 8504 Géographie-cités, berroir@paris7.jussieu.fr

\section{CLAUDE GRASLAND}

UMR 8504 Géographie-cités, Université Paris 7/UFR GHSS, claude.grasland@parisgeo.cnrs.fr

\section{FRANCE GUÉRIN-PACE}

INED, Paris, guerin@ined.fr

\section{GRÉGORY HAMEZ}

Université de Metz (CEGUM), hamez@univ-metz.fr 